\title{
Chronic pelvic pain and the role of exploratory laparoscopy as diagnostic and therapeutic tool: a retrospective observational study
}

\author{
Géraldine Brichant ${ }^{1}$, Marie Denef ${ }^{1}$, Linda Tebache ${ }^{1}$, Gaëlle Poismans', Serena Pinzauti ${ }^{2}$, Valérie Dechenne ${ }^{1}$
} and Michelle Nisolle 1* $^{*}$

\begin{abstract}
Background: Forty percent of exploratory laparoscopies are performed for chronic pelvic pain (CPP). However, a final diagnosis is still unreported in $35 \%$ of the patients. We decided to evaluate the identification of pathological lesions and the improvement of painful symptoms in patients with CPP and normal physical examination and imaging and who are scheduled for exploratory laparoscopy. The prospective study was designed in a tertiary referral center for endometriosis. Forty-eight patients complaining of CPP and scheduled for exploratory laparoscopy were included. Pelvic pain intensity was assessed using the visual analogue pain scale (VAS), and at inclusion, negative clinical and imaging assessments were required. During exploratory laparoscopy, the recognized lesions were reported and different surgical treatment options were performed depending on the location of the lesion.

Results: In 98\% of the cases, exploratory laparoscopy demonstrated the presence of pelvic anomalies that had not been diagnosed at the time of clinical and imaging examination. After surgery, a significant improvement of CPP has been demonstrated in 24 (59\%) patients with VAS $<5$ postoperatively.

Conclusions: Exploratory laparoscopy is reasonable in patients complaining of CPP, allowing a final diagnosis in a high percentage of patients and a significant improvement in pain symptom in 59\% of the cases. This study was retrospectively registered by our local Ethics Committee on February 7, 2018 (B412201835729).
\end{abstract}

Keywords: Chronic pelvic pain, Exploratory laparoscopy, Endometriosis, Excision of uterosacral ligaments

\section{Background}

Chronic pelvic pain (CPP) is defined as intermittent or constant pain lasting since at least 6 months in the lower abdomen or the pelvis. It can be localized in the pelvis, the anterior abdominal wall at the umbilicus or below, and the lumbosacral back or the buttocks and is sufficient to cause functional disability or lead to seek medical care [1]. Almost $15 \%$ of women between 18 and 49 years old complain of CPP, but less than a third seek medical advice [2]. CPP is responsible for about $10 \%$ of gynecological

\footnotetext{
* Correspondence: michelle.nisolle@chrcitadelle.be

${ }^{1}$ University Department of Obstetrics and Gynecology, CHR La Citadelle,

Boulevard du Douzième de Ligne, 1, 4000 Liège, Belgium

Full list of author information is available at the end of the article
}

consultations and represents the surgical indication of $40 \%$ of exploratory laparoscopies [1]. CPP may be related to different causes, from gynecological diseases to gastro-intestinal and urological pathologies. Although less common in such patients, neurological, musculoskeletal, and psychological diseases should be considered [3]. In 25 to $50 \%$ of the cases, more than one anomaly can be found in a single patient, increasing the difficulties in diagnosing and alleviating the symptoms [4]. A full medical history, associated with a complete medical examination, is key in order to address patients' correct diagnosis and management. Nowadays, it becomes more and more obvious that a multidisciplinary approach is one of the best way to help the patient in an individualized manner [5]. 
Considering our experience, we found that patients' history is often characterized by a long series of medical advices and wrong diagnoses before the final treatment.

The present study aims to establish if exploratory laparoscopy demonstrates the presence of pathological lesions in patients with normal physical and complementary examinations complaining of $\mathrm{CPP}$ and to evaluate the improvement of pain after the surgical procedures.

\section{Methods}

This study took place in a tertiary referral Center for endometriosis, in the University department of Obstetrics and Gynecology of Liège, Belgium, between October 2011 and April 2015. A total of 48 patients complaining of CPP and scheduled for a surgical treatment were included in the present study following guidelines of our Ethics Committee (B412201835729, retrospectively registered February 7, 2018).

In order to evaluate the role and the efficacy of exploratory laparoscopy in patients with CPP, women in reproductive age (range 18-45 years old) with CPP and negative clinical and imaging examination were considered as possible candidates in the study. CPP was assessed using a 10-point visual analogue pain scale (VAS), and only women with a VAS $\geq 8$ were included in the study. Negative clinical examination or only a uterosacral ligament (USL) thickening or uterine retroversion were considered as inclusion criteria. All patients had had a negative ultrasound from their referent OB-GYN. Negative imaging assessments, such as barium enema, pelvic computerized tomography $(\mathrm{CT})$, or pelvic magnetic resonance imaging (MRI), were also required at inclusion in the study. Exclusion criteria were an evidence of chronic disease at medical history or anatomic or endometriotic lesion on clinical examination and imaging assessments.

At inclusion, VAS score for chronic pelvic pain was noted for each patient. During exploratory laparoscopy, a complete examination of the abdominal and pelvic cavity was performed. The observed lesions were classified as endometriosis, thickening of USLs with suspicion of endometriosis, adhesions, uterine anomalies, adnexal anomalies, or any other anomalies. Patients had been counseled before surgery about the different treatment options offered: excision of endometriosis, excision of torus uterinum, adhesiolysis, total hysterectomy with or without bilateral salpingo-oophorectomy (SO), salpingectomy, oophorectomy, or any possible surgical treatment. More than one lesion could be observed in each patient, and subsequently more than one surgical procedure, performed. All specimens were sent for histological examination.

After surgery, VAS score for chronic pelvic pain was assessed at 3- and 24-month interval during consultations.
Depending on the VAS score, patients were divided into the following four groups: group A: VAS 0, no pain; group B: VAS 1-4, mild pain; group C: VAS 5-7, moderate pain; and group D: VAS 8-10, severe pain.

\section{Statistical analysis}

Data were collated in a secured data file and were analyzed thanks to the Excel software. Results were expressed as mean \pm standard deviation for continuous variable or as percentages for discrete variables. A $p$ value $<0.05$ was considered as statistically significant.

\section{Results}

The characteristics of the patients are summarized in Table 1. Data were analyzed in 41 patients as 6 patients were lost to follow-up, and in one case, laparoscopy was unsuccessful due to severe adhesions secondary to bowel resection for necrotizing enterocolitis in infancy, leading to unacceptable surgical risks. At exploratory laparoscopy, the documented lesions were reported as follows: 19 (46\%) endometriotic lesions such as deep infiltrating endometriosis nodule or peritoneal lesions; 19 (46\%) endometriotic suspected lesion (USL thickening); 14 (31\%) abdomino-peritoneal adhesions and adhesions between the adnexa and bowel or uterus and bladder; 5 (12\%) uterine anomalies (increased uterine volume, abnormal vascularization, suspicion of adenomyosis); 7 (17\%) adnexal anomalies (non-dilated hydrosalpinx, ovarian cyst of small size); and $1(2 \%)$ normal pelvis (Fig. 1 and Table 2). In summary, exploratory laparoscopy demonstrated the presence of pelvic anomalies not previously detected in $98 \%$ of patients.

Considering surgical procedures, we performed excisions of visible endometriosis 17 patients (41\%); excisions of USLs in 24 patients (59\%); adhesiolysis in 10 patients (24\%); total hysterectomies (1 with unilateral SO and 4 without) in 5 patients (12\%); cystectomy, salpingectomies, $\mathrm{SO}$, or other procedures on the adnexa in 7 patients (17\%); excision of the post-hysterectomy vaginal scar in 2

Table 1 Characteristics of patients

\begin{tabular}{ll}
\hline Characteristics & Patients \\
\hline Age & $\begin{array}{l}\text { Range 18-45 years old; } \\
\text { mean 32 years old }\end{array}$ \\
VAS score & Mean 8.8/10 ( \pm 0.9$)$ \\
Complementary examinations & \\
• Barium enema & $27 \%$ \\
• Colonoscopy & $5 \%$ \\
• Pelvic computerized tomography (CT) & $17 \%$ \\
• Pelvic magnetic resonance imaging (MRI) & $68 \%$ \\
Medical history of abdomino-pelvic surgery & \\
• Endometriosis & $17 \%$ \\
- Other abdomino-pelvic surgery & $56 \%$ \\
Parity & $46 \%$ nulliparous \\
\hline
\end{tabular}




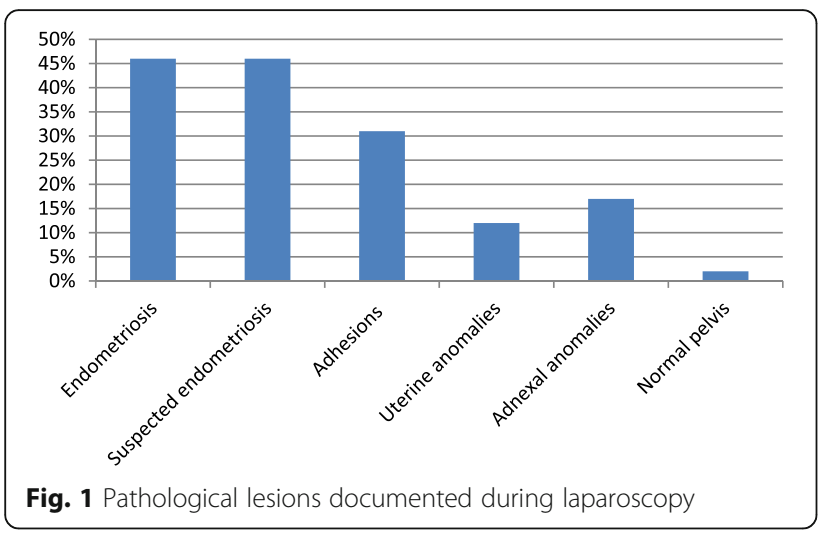

patients (5\%); and treatment of uterine retroversion in 1 patient (2\%). In one patient (2\%), no surgical procedure was performed (Fig. 2 and Table 2). As previously mentioned, more than one lesion could have been described in a patient and subsequently more than one surgical procedure would then be performed. In all cases but one, adhesiolysis has been always associated with another surgical procedure. In two thirds of the patients undergoing excision of USLs, another procedure was performed. No intraoperative complications occurred during surgeries, and no abdominal conversion to laparotomy was needed.

The histological examination confirmed the presence of the corresponding pathological diseases in four cases (24\%) of macroscopic endometriosis, in nine cases (38\%) of suspected endometriosis (USL thickening), in five cases $(100 \%)$ of uterine anomalies after hysterectomies (adenomyosis, salpingitis, leiomyomas, endometriosis), and in three cases (43\%) of adnexal anomalies (Fig. 2).

Postoperative pain was assessed, and patients were divided into four groups as previously mentioned: 18

Table 2 Surgical procedures and number of patients improved depending on the procedure

\begin{tabular}{lll}
\hline Surgical procedures & $\begin{array}{l}\text { Number of patients: } \\
\mathrm{n}(\%)\end{array}$ & $\begin{array}{l}\text { Number of patients } \\
\text { improved (\%) }\end{array}$ \\
\hline Visible endometriosis & $17(41 \%)$ & $9(53 \%)$ \\
USLs excisions & $24(59 \%)$ & $13(54 \%)$ \\
Adhesiolysis & $10(24 \%)$ & $6(60 \%)$ \\
Total hysterectomies & $\begin{array}{l}5(12 \%) \\
(1 \text { with unilateral SO) }\end{array}$ & $5(100 \%)$ \\
$\begin{array}{l}\text { Other adnexal procedures: } \\
\text { - Cystectomy }\end{array}$ & $7(17 \%)$ & $5(71 \%)$ \\
$\cdot$ Salpingectomy & & \\
- SO & & \\
- others & & $1(50 \%)$ \\
$\begin{array}{l}\text { Excision of post } \\
\text { Uterectomy scar }\end{array}$ & $2(5 \%)$ & $1(100 \%)$ \\
Uterine retroversion & $1(2 \%)$ & $0(0 \%)$ \\
treatment & & \\
Nothing & $1(2 \%)$ &
\end{tabular}

patients (44\%) had no residual pain (VAS score 0), 6 patients (15\%) had mild pain (VAS score between 1 and 4), 8 patients (19\%) had moderate pain (VAS score between 5 and 7 ), and 9 patients (22\%) had severe pain with no improvement of CPP and with VAS score $\geq 8$ (Fig. 3).

Considering the single surgical procedures only, at the time of endometriotic lesion resection, 10 (53\%) patients reported a significant improvement of pain (group A and group B), while excision of USLs, adhesiolysis, cystectomy or salpingectomies or SO, and hysterectomy led to a considerable improvement of pain in 13 (54\%), 6 (60\%), 5 (71\%), and $5(100 \%)$ of cases, respectively (Fig. 4 and Table 2).

\section{Discussion}

Chronic pelvic pain is a debilitating condition among women with a major impact on health-related quality of life, on work productivity, and on the health care system and concerns about $4 \%$ of women [1]. Identifying the origin of CPP is difficult as it may be caused by disorders of the reproductive tract, gastrointestinal system, urological organs, and musculoskeletal and psychoneurological systems [6]. Some conditions are often associated in the same patient, and many factors have to be evaluated and be taken care of. Pelvic lesions discovered during exploratory laparoscopy are not necessarily responsible for the pain described by the patient [2]. The medical history and physical examination of the patient are essential to allow an appropriate management of the disease.

Gynecologic conditions account for approximately $20 \%$ of cases of CPP [3, 7], and irritable bowel syndrome (IBS) and interstitial cystitis (IC) are the other most diagnosed pathology [8]. Considering the different specific gynecological pathologies, several studies describe endometriosis and adhesions as the most frequent causes of CPP (80 and 52\%, respectively) [1, 2, 9]. In our series, the high incidence of endometriosis and adhesions in patients with CPP was confirmed, accordingly with previous results.

However, if the association between painful symptoms and endometriosis or adhesions is well accepted, the precise causal relationship is still poorly understood. Endometriosis is still found in $2-50 \%$ of asymptomatic women [4], confirming that the scientific research in this field remains a priority. The three most commonly suggested mechanisms for pain in endometriosis are the production of chemokines (growth factors and cytokines), the direct and indirect effects of active bleeding from endometriotic implants, and the irritation or direct invasion of pelvic floor nerves by infiltrating endometriotic lesions [10]. Central sensitization is also a mechanism involved in the pain process in patients with endometriosis. Becker et al. showed in their review that central changes might explain why pain could become more and more difficult to treat 


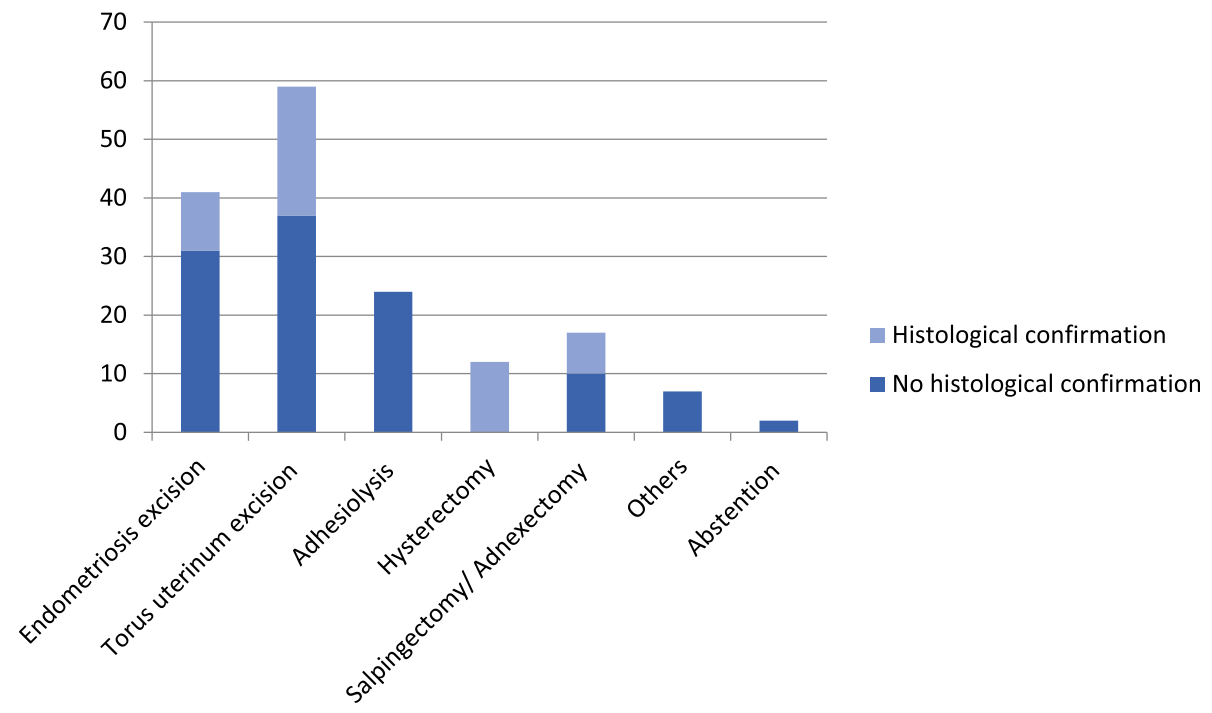

Fig. 2 Surgical procedures performed during laparoscopy

despite appropriate medication. This could also explain the gap sometimes existing between the extent of the disease and the importance of the pain and/or the persistence of the pain despite extensive surgery [11]. However, as the authors stated, further studies targeting central changes in women with proven endometriosis and their influence on pain symptoms and the response to treatment are needed.

Nowadays, the relationship between deep infiltrating endometriosis (DIE) and painful symptoms is well consolidated, even if the extent of lesions does not correlate with the severity of pain [12]. On the contrary, no strong evidence can be found for a relation between endometrioma and painful symptoms. Two reports suggested that painful endometriomas are frequently associated with pelvic adhesions, peritoneal implants, or deep infiltrating lesions, and the severity of pain is independent from the size of the endometrioma $[13,14]$.

Endometriotic lesions may have variable appearance. The histological distinction in peritoneal, ovarian, and deep-infiltrating endometriosis as three separate entities has been well established [15]. Endometriotic lesions

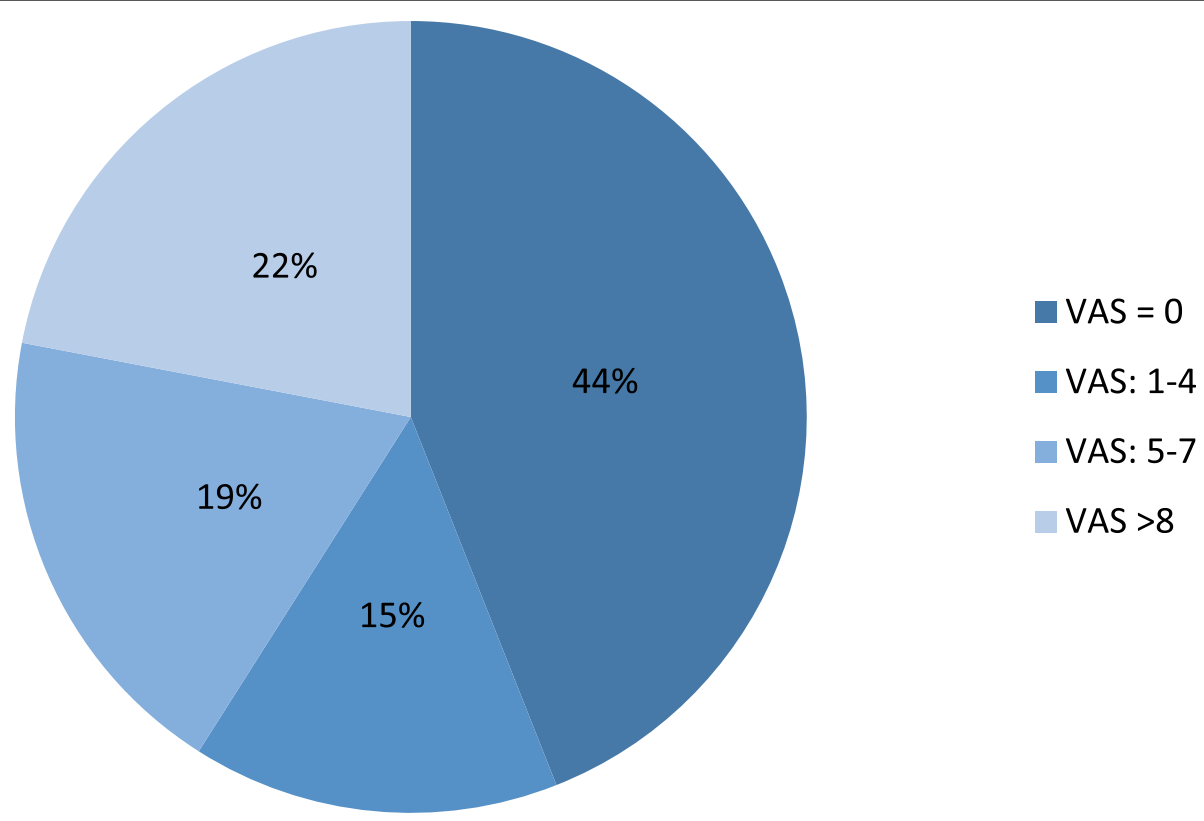

Fig. 3 Pain improvement after surgery 


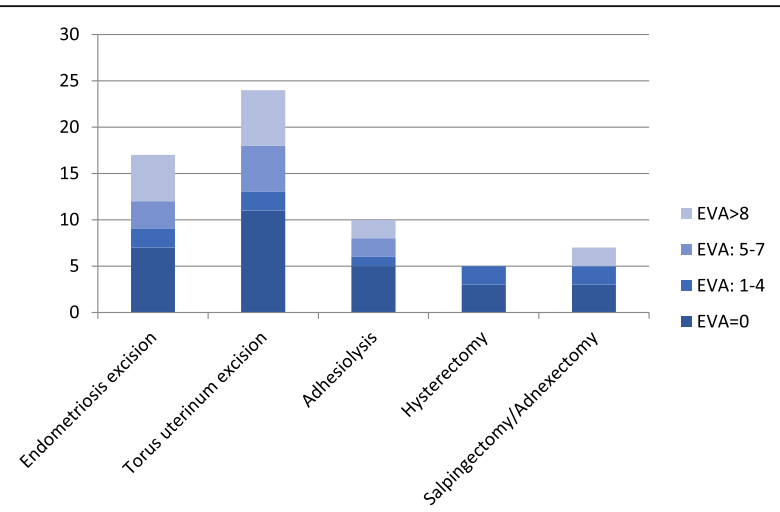

Fig. 4 Pain postoperative improvement depending on the surgical treatment

may have typical aspects, purple or blue nodules, or more atypical presentations in $15-30 \%$ of patients (peritoneum opacification or circular defects, glandular growths, inflammatory alterations, yellow spots, ovarian adhesions) that may be confused with other non-endometriotic lesions [16]. Even if the gold standard for endometriosis diagnosis remains the exploratory laparoscopy, this technique in unexperienced hands may still ignore some atypical or retroperitoneal endometriotic lesions. Nisolle et al. also showed that microscopic endometriosis, invisible at the laparoscopic assessment, can be diagnosed during anatomopathological analysis [17]. The old concept of microscopic endometriosis in visually normal peritoneum reported more than a decade ago and supported at that time by few series was recently confirmed by Khan et al. [18]. Clinical consequences of this endometriosis are unknown. However, some of the most recent papers studying microscopic intestinal endometriosis tend to be giving those tiny lesions some clinical relevance. Authors have proved the presence of microscopic endometriosis at a distance from macroscopic lesions that are resected. In those cases, radical segmental colectomy would not be more efficient than discoid resection as it would not remove the microscopic lesions [19, 20].

In summary, the increasing knowledge of the variability of endometriotic lesions appearance has led to a significant increase in endometriosis diagnosis. However, a high incidence of indefinite diagnosis is still reported in patients with CPP as no visible pathology can be found in $35 \%$ of cases [9]. This proportion of failure to diagnose an etiology for the CPP is high considering that exploratory laparoscopy remains an invasive surgical procedure with the inherent risks. In our series, the rate of negative exploratory laparoscopy is extremely low (2\%) and this data may be explained by the fact that all patients had been carefully examined and investigated by a gynecologist specialized in CPP and endometriosis. The same experimented gynecologist performed all surgical procedures. From data about the lesions' appearance, the development of other surgical procedures arises, such as the torus uterinum excision (or resection of uterosacral ligament(s)). Surgery is performed by removing all the USLs at the pararectal levels until their insertions on the cervix. This technique should be differentiated from the laparoscopic uterosacral nerve ablation (LUNA), consisting in a single uterosacral ligament section at $1-2 \mathrm{~cm}$ from their insertion on the cervix, and the pre-sacral neurectomy (PSN), consisting in the interruption of the sympathetic nervous fibers at the superior hypogastric plexus. Both LUNA and PSN are currently considered non-specific procedure for CPP and are no longer recommended of these patients [9]. In our experience, torus uterinum excision has been performed in patients whose USLs were tight and sore on clinical examination and/or if their thickened appearance was confirmed at the exploratory laparoscopy. More than half of our patients described less pain after the surgery even if their satisfaction was not always related to the histological confirmation of endometriosis. This procedure was efficient in $54 \%$ (group A and B) even if the presence of endometriosis was confirmed only for 5 patients on 13 (38\%).

Our data on histological examination confirmed the presence of endometriosis in only $24 \%$ of treated patients for macroscopic endometriosis and in $38 \%$ of patients with USLs excision. Actually, negative histological examination cannot exclude endometriosis diagnosis because either all rigorous histological criteria might not be met or lesions might not visible because of hormonal suppression or histological exploration might be incomplete (i.e., small lesions surrounded by large sections of healthy tissue). There is no consensus about technics for histological examination. Histological examination is recommended but a negative histological examination cannot exclude endometriosis diagnosis [21, 22].

Considering adhesions, as previously reported, they are frequently related to the endometriotic process and may be actually considered as a possible cause of pain-related endometriosis. Adhesions may theoretically be caused by other pelvic inflammatory processes, such as pelvic inflammatory disease, inflammatory bowel disease, and previous abdominal-pelvic surgery but in 50\% of cases the etiology is unknown [9]. The relationship between adhesions and CPP is controversial. Usually, studies show a similar frequency of adhesions between women with CPP and without CPP [9]. However, some series report some solid arguments in favor of a role of adhesions in CPP. Even as early as the 1980s, Kresch found a higher frequency of adhesions in women with CPP compared with women without CPP. In his study, he noted a characteristic aspect of adhesions in women with CPP that appeared to be restricting the motion or the expansibility of one or more organs [23]. More 
recently, the role of adhesions seemed to be confirmed by the laparoscopy conscious pain mapping where a laparoscopy is performed under local anesthesia. The study showed that under local anesthesia stimulation of adhesions elicited pain in patients [24]. In our series, adhesions were described in $31 \%$ of patients with CPP and we performed 10 (24\%) of adhesiolysis with a significant improvement of pain symptomatology (groups A and B) in $60 \%$ of treated patients. Except in one case, this procedure has been always associated to another surgical procedure and it seems difficult to make a judgment on its benefit.

CPP represents $12 \%$ of all the indications for hysterectomy [25]. A recent review tells that in the absence of any obvious pathology, 60 to $79 \%$ of women report improvement in symptomatology after hysterectomy. In our series, we performed four $(10 \%)$ total hysterectomies and one (2\%) total hysterectomies with BSO when the uterus or adnexa had abnormal aspect, such as increased volume, and abnormal vascularisation or fibroid appearance. Histological examination confirmed pathologies such as adenomyosis, endosalpingiosis, salpingitis, endometritis, and leiomyoma in $100 \%$ of the specimen. All patients had significant improvement of pain after the procedure. Our data are similar to those of the literature, confirming that hysterectomy may be effective against pain, even in the absence of obvious anomalies of the uterus. However, it should be important to inform patients that in 21 to $40 \%$ of the cases the intervention will not help in improving CPP and rarely will it worsen the painful symptomatology. Hysterectomy should be categorized as a non-specific treatment. It should be kept in mind that 70\% of CPP are non-gynecologic etiology such as irritable bowel syndrome, painful bladder syndrome, and pelvic floor myalgia. All women with CPP should undergo a multidisciplinary evaluation before surgery to exclude other causes of pain to maximize the chances of pain resolution [25].

We performed five (12\%) interventions at adnexal level with one cystectomy and one salpingo-oophorectomy for cyst and three salpingectomies. Pelvic inflammatory disease is found in 5\% of laparoscopy performed to CPP. One fourth of women who had acute salpingitis will develop CPP. The aftereffect of acute salpingitis can be variable and include adhesions, hydrosalpinx, and ovarian dystrophi [2]. Ovarian cysts are found in 3\% of laparoscopy performed for CPP. Ovarian cysts are usually asymptomatic or cause acute pain. Recurrent functional ovarian cysts seem to be sometimes the cause of CPP. In our series, adnexal surgeries have been associated to another procedure except in one case of the adnexectomy for a cyst. In all those cases, the patient is pain free (group A).

\section{Conclusion}

In conclusion, our study demonstrated that exploratory laparoscopy provides a definitive diagnosis in $98 \%$ of women complaining of unexplained CPP. The surgical treatment of these lesions improves painful symptomatology in $59 \%$ of women with a total disappearance of pain in $44 \%$ of cases (VAS 0) and significant improvement in $15 \%$ of cases (VAS < 5). We believe that the exploratory laparoscopy is therefore justified in patients complaining of significant CPP. The candidates' selection should be meticulous, and patients should be investigated at urologic, gastroenterologic, and musculoskeletal level before surgery. We believe that women should be referred to experimented gynecologists in the field of CPP and endometriosis who can properly inspect the pelvic area and who are able to detect lesions potentially responsible of CPP. Despite that, $22 \%$ of women do not improve after surgery. This could either be because of the found anomaly wasn't the only one responsible for the painful symptoms and/or that normal anatomy couldn't be restored completely. We believe a better candidates' selection including the identification of poor prognostic factor such as psychosomatic syndrome could improve the results as well as a better identification of macroscopic and microscopic conditions. A better understanding of relationship between some type of lesions and CPP will help in alleviating the patients' symptoms.

\section{Abbreviations \\ CPP: Chronic pelvic pain; CT: Computerized tomography; DIE: Deep infiltrating endometriosis; IBS: Irritable bowel syndrome; IC: Interstital cystitis; LUNA: Laparoscopic uterosacral nerve ablation; MRI: Magnetic resonance imaging; PSN: Pre-sacral neurectomy; SO: Salpingo-oophorectomy; USLS: Utero-sacral ligaments; VAS: Visual analogue pain scale}

\section{Acknowledgements}

Special thanks to Marie Timmermans for her help in the data collection and analysis.

Availability of data and materials

Please contact author for data requests.

Authors' contributions

$\mathrm{GB}, \mathrm{MD}, \mathrm{GP}$, and SP have collected the data. MN and VD performed the surgeries. GB, MD, and MN wrote the manuscript. All authors read and approved the final manuscript.

Ethics approval and consent to participate

This study was conducted according to the Ethical Guidelines of our institution, and no consent to participate was needed (Ethics Committee 412, CHR Citadelle, Liege, Belgium (study registration number: B412201835729)).

\section{Consent for publication}

Not applicable

\section{Competing interests}

The authors declare that they have no competing interests.

\section{Publisher's Note}

Springer Nature remains neutral with regard to jurisdictional claims in published maps and institutional affiliations.

\section{Author details}

'University Department of Obstetrics and Gynecology, CHR La Citadelle, Boulevard du Douzième de Ligne, 1, 4000 Liège, Belgium. ${ }^{2}$ UO Ostetricia e Ginecologia, Ospedale Santa Maria alla Gruccia, Arezzo, Montevarchi, Italy. 
Received: 12 April 2018 Accepted: 20 June 2018

Published online: 20 July 2018

\section{References}

1. Howard FM (2003) Chronic pelvic pain. Obstet Gynecol 101:594-611

2. Fauconnier A, Fritel X (2010) Algies pelviennes chroniques d'origine non endométriosique. J Gynecologie, Obstetrique et Biologie de la Reproduc 39: s1-S342

3. Steege JF, Siedhoff MT (2014) Chronic pelvic pain. Obstet Gynecol 124:616629. https://doi.org/10.1097/AOG.0000000000000417

4. Moen MH, Stokstad T (2002) A long-term follow-up study of women with asymptomatic endometriosis diagnosed incidentally at sterilization. Fertil Steril 78:773-776

5. Morrissey D, Ginzburg N, Whitmore K (2014) Current advancements in the diagnosis and treatment of chronic pelvic pain. Curr Opin Urol 24:336-344. https://doi.org/10.1097/MOU.0000000000000062

6. Alcock A (1926) Chronic pelvic pain in women. Br Med J 1:609-612

7. Zondervan KT, Yudkin PL, Vessey MP, Dawes MG, Barlow DH, Kennedy SH (1999) Patterns of diagnosis and referral in women consulting for chronic pelvic pain in UK primary care. Br J Obstet Gynaecol 106:1156-1161

8. Vercellini P, Somigliana E, Vigano P, Abbiati A, Barbara G, Fedele L (2009) Chronic pelvic pain in women: etiology, pathogenesis and diagnostic approach. Gynecol Endocrinol 25:149-158. https://doi.org/10.1080/ 09513590802549858

9. Howard FM (2000) The role of laparoscopy as a diagnostic tool in chronic pelvic pain Bailliere's best practice \& research. Clinical Obstetrics \& Gynaecology 14:467-494

10. Berkley KJ, Rapkin AJ, Papka RE (2005) The pains of endometriosis. Science 308(5728):1587-1589. https://doi.org/10.1126/science.1111445

11. Morotti M, Vincent $K$, Becker CM (2017) Mechanisms of pain in endometriosis. Eur J Obstet Gynecol Reprod Biol. 209:8-13. https://doi.org/ 10.1016/j.ejogrb.2016.07.497

12. Fauconnier A, Chapron C, Dubuisson JB, Vieira M, Dousset B, Breart G (2002) Relation between pain symptoms and the anatomic location of deep infiltrating endometriosis. Fertil Steril 78:719-726

13. Fauconnier A, Chapron C (2005) Endometriosis and pelvic pain: epidemiological evidence of the relationship and implications. Hum Reprod Update 11(6):595-606. https://doi.org/10.1093/humupd/dmi029

14. Khan KN, Kitajima M, Fujishita A, Hiraki K, Matsumoto A, Nakashima M, Masuzaki H (2013) Pelvic pain in women with ovarian endometrioma is mostly associated with coexisting peritoneal lesions. Hum Reprod 28:109-118. https://doi.org/10.1093/humrep/des364

15. Nisolle M, Donnez J (1997) Peritoneal endometriosis, ovarian endometriosis, and adenomyotic nodules of the rectovaginal septum are three different entities. Fertil Steril 68:585-596. https://doi.org/10.1016/S00150282(97)00191-X

16. Lamvu G, Tu F, As-Sanie S, Zolnoun D, Steege JF (2004) The role of laparoscopy in the diagnosis and treatment of conditions associated with chronic pelvic pain. Obstet Gynecol Clin N Am 31:619-630. https://doi.org/ 10.1016/j.ogc.2004.05.003

17. Nisolle M, Paindaveine B, Bourdon A, Berliere M, Casanas-Roux F, Donnez J (1990) Histologic study of peritoneal endometriosis in infertile women. Fertil Steril 53:984-988

18. Khan KN, Fujishita A, Kitajima M, Hiraki K, Nakashima M, Masuzaki H (2014) Occult microscopic endometriosis: undetectable by laparoscopy in normal peritoneum. Hum Reprod 29:462-472. https://doi.org/10.1093/humrep/det438

19. Badescu A et al (2016) Mapping of bowel occult microscopic endometriosis implants surrounding deep endometriosis nodules infiltrating the bowel. Fertil Steril 105(2):430-434. https://doi.org/10.1016/j.fertnstert.2015.11.006

20. Darwish B, Roman H (2016) Surgical treatment of deep infiltrating rectal endometriosis: in favor of less aggressive surgery. Am J Obstet Gynecol 215(2):195-200. https://doi.org/10.1016/j.ajog.2016.01.189

21. Fritel X (2007) Endometriosis anatomoclinical entities. J Gynecologie, Obstetrique et Biologie de la Reproduc 36:113-118. https://doi.org/10.1016/ j.jgyn.2006.12.003

22. Kennedy $S$ et al (2005) ESHRE guideline for the diagnosis and treatment of endometriosis. Hum Reprod 20:2698-2704. https://doi.org/ 10.1093/humrep/dei135

23. Kresch AJ, Seifer DB, Sachs LB, Barrese I (1984) Laparoscopy in 100 women with chronic pelvic pain. Obstet Gynecol 64:672-674
24. Howard FM, El-Minawi AM, Sanchez RA (2000) Conscious pain mapping by laparoscopy in women with chronic pelvic pain. Obstet Gynecol 96:934-939

25. Lamvu G (2011) Role of hysterectomy in the treatment of chronic pelvic pain. Obstet Gynecol 117:1175-1178. https://doi.org/10.1097/AOG. ob013e31821646e1

\section{Submit your manuscript to a SpringerOpen ${ }^{\circ}$ journal and benefit from:}

- Convenient online submission

- Rigorous peer review

- Open access: articles freely available online

- High visibility within the field

Retaining the copyright to your article

Submit your next manuscript at $>$ springeropen.com 